\title{
COMPONENTES DEL CRECIMIENTO Y REDISTRIBUCION DE LA POBLACIÓN MEXICANA: IMPLICACIONES DE LOS RESULTADOS PRELIMINARES DEL CENSO DE 1980
}

\author{
WILLIAM W. WINNIE \\ Centro de Investigaciones Sociales y Económicas \\ Facultad de Economía - Universidad de Guadalajara
}

LOS ReSUltados preliminares del X Censo General de Población se limitan a un primer informe sobre el número de habitantes, por sexo, de cada entidad federativa y del país en general. Aunque están sujetos a reajustes en etapas posteriores del proceso censal, el Consejo Nacional de Población considera que las diferencias entre ellos y los datos definitivos serán muy pequeña (CONAPO, 1980).

No obstante esta limitación, es posible realizar comparaciones con algunos datos de los cuatro censos previas a éste, con las estadísticas, vitales y otros conocimientos existentes en la actualidad (entre ellos, los referentes al impacto de las migraciones internacionales) que permiten elaborar conclusiones provisorias acerca del crecimiento y la redistribución de la población mexicana durante la última década. El presente trabajo encara los siguientes temas, respecto al número total de habitantes (sin tomar en consideración la variable sexo, para la cual existen datos semejantes):

1. Componentes del crecimiento a nivel nacional en el periodo 1970-80;

2. Redistribución interna de la población mexicana durante el último medio siglo y, en particular, a partir de 1970;

3. Componentes de la redistribución interna: conclusiones y especulaciones basadas en datos incompletos;

4. Implicaciones para la política demográfica del país.

Es, entonces, un trabajo técnico que no pretende llegar a interpretaciones teóricas de los hechos: su propósito se restringe a efectuar una evaluación preliminar de los primeros resultados del censo de 1980 frente a otros datos y a analizar sus implicaciones en términos de tendencias demográficas recientes.

Cabe insistir, para beneficio de posibles lectores que no sean especialistas en materia de redistribución de la población mexicana, que existen ciertas limitaciones en los datos básicos que utiliza el presente trabajo. Entre ellas, 
ya se mencionó el carácter preliminar de la información disponible del censo de 1980 y su muy restringido alcance temático. Hay otras limitaciones que también son importantes.

En primer término, los censos mexicanos de 1960 y anteriores - como es usual en todos los censos nacionales- se vieron afectados (de forma demostrable) por fuertes omisiones de datos en la franja que consigna a los niños pequeños. Es muy probable que los datos definitivos (y desglosados) del censo de 1980 muestren lo mismo respecto al de 1970 y que, a su vez, ellos también estén sujetos a omisiones similares. También es probable que la tasa de estas omisiones varie de modo más o menos notables entre estados y entre censos.

En segundo lugar, demógrafos calificados que trabajan con esta información consideran poco confiables las estadísticas vitales del país, en parte debido a la gran demora en el registro de muchos eventos $y$, hasta hace pocos años por su consignación exclusiva en base al lugar de ocurrencia. Aunque estas consideraciones podrían parecer simples tecnicismos, los demógrafos son conscientes de que sus efectos sobre los datos registrados a nivel de estados, pueden revestir una considerable importancia.

Por otra parte, si el objetivo es analizar los efectos de las migraciones internacionales a corto, mediano y largo plazo se corre el riesgo de caer en el reino de las estimaciones burdas (en el mejor de los casos). En general, la información que existe sobre el aspecto cuantitativo del fenómeno varía de especulaciones interesantes realizadas por científicos sociales desapasionados (hechas en base a información disponible) hasta abultadas presentaciones de cifras hechas por autores que en muchos casos escriben en tono polémico que aparentemente están en su totalidad fuera de los límites de lo posible. A nivel cualitativo existe un gran caudal de conocimientos fundamentados cieníficamente que demuestra que estos movimientos constituyen un factor importante en el crecimiento demográfico nacional, y que sus efectos varían ampliamente entre zonas diferentes del país; pero ni la magnitud general ni las variaciones entre estados pueden cuantificarse en la actualidad con precisión.

Estas consideraciones imponen limites muy severos a las inferencias cuantitativas que puedan hacerse sobre los temas que se contemplan más adelante. El presente trabajo procura elaborar un conjunto de conclusiones de tipo cualitativo, que comparativamente posean un alto grado de confiabilidad - a pesar de las dudas que existen respecto de la cuantificación de muchos aspectos importantes.

\section{Componentes del crecimiento nacional, 1970-80}

La población residente de la República Mexicana creció a un ritmo anual medio de 3.289 por ciento, entre el censo de 1970 y el de $1980 .^{\prime}$ Contribuyeron a esta alta tasa general de crecimiento, la reproducción natural —que

\footnotetext{
1 Los datos censales de 1980 son preliminares y los de censos anteriores son definitivos. Para el periodo intercensal $1970-80$, se usó $n=3777 / 365$ en los cálculos del presente trabajo, cuando $P_{x t}=P_{70}(i+r)^{\mathrm{h}}$. En forma semejante, se usó $n=3519 / 365$ para el período $1960-70$.
} 
todavía está entre las más rápidas del mundo a nivel nacional-y, en forma negativa, una emigración neta (de magnitud significativa pero no cuantificable) en las migraciones internacionales.

\section{Las migraciones internacionales}

México tiene una larga historia como "exportador" de población. Estos movimientos se dirigen en primer término hacia los Estados Unidos, y son de tal magnitud que los intercambios poblacionales netos con otros países son, en comparación, tan pequeños que no revisten importancia para los fines del presente trabajo.

Diversos autores han demostrado, en base a trabajos directos en muchas comunidades de origen, que la gran mayoría de los mexicanos que se trasladan a los Estados Unidos lo hacen para obtener mayores ingresos durante un lapso relativamente corto, para regresar a sus lugares de origen después de haber trabajado algunas semanas o meses o, con frecuencia mucho menor, dos o tres años (para una bibliografía al respecto, ver Winnie Arroyo y Guzmán, 1980: citas 505-628). Muchos, sin embargo, van por plazos más largos: si bien no a quedarse alli definitivamente, al menos a pasar el resto de su vida económicamente activa en trabajos que les permiten niveles materiales de vida mucho más elevados que los que podrían disfrutar en su país de origen. En años recientes, los migrantes "ilegales" o "indocumentados" obviamente forman el grueso del primer grupo; mientras que la mayoria de los de más largo plazo, al parecer, "se documentan" legalmente después de alguna experiencia previa como "indocumentados" (ver, por ejemplo, Alba, 1978; Hirschman, 1978).

Debido al peso significativo que poseen los movimientos laborales de corto plazo dentro de la totalidad de los intercambios migratorios entre México y los Estados Unidos, a la falta de nitidez en la distinción entre movimientos de corto y largo plazo además del hecho de que muchos de los movimientos, por ser de "indocumentados" no se registran en las estadísticas de ninguno de los dos países, no es posible cuantificar la migración neta de mexicanos hacia los Estados Unidos. ${ }^{2}$

Lo que parece ser seguro y ampliamente aceptado por los estudiosos que se dedican al tema es lo siguiente: con excepción de breves lapsos de recesión (o depresión) económica en los Estados Unidos, la migración neta de mexicanos hacia ese pais ha sido comparativamente intensa durante la mayor parte del siglo $x \mathrm{X} y$, en general, ha mostrado un ritmo creciente a largo plazo: sin embargo no es posible cuantificar adecuadamente el movimiento neto para cualquier periodo, aunque no queda lugar a dudas de que el saldo ha sido de signo negativo para el crecimiento de la población mexicana, en lo que va del siglo.

2 Los intentos para cuantificar estos movimientos casi llegaron al nivel de ser el "deporte favorito" entre los cientificos sociales interesados en el tema durante los años setenta. El título de "Counting the uncountable: Estimates of undocumented aliens in the United States" usado por Keely (1977) en uno de sus trabajos es muy sugerente al respecto. 


\section{El crecimiento natural}

Si se acepta en principio que el saldo migratorio internacional fue un factor negativo en el crecimiento de la población mexicana durante el período intercensal 1970-80, es necesario descartar de inmediato los datos de las estadísticas vitales al respecto. Las tasas de crecimiento natural de 3.25 por ciento por año reportados para 1970 y 3.20 para 1976 (CONAPO, 1979a: cuadro IV-2), son incongruentes con los datos censales que muestran un crecimiento anual medio de $\mathbf{3 . 2 8 9}$ por ciento entre los dos censos: el crecimiento natural medio debe haber sido de por lo menos 3.3 por ciento durante el decenio, lo cual implicaría una emigración neta de apenas 7300 mexicanos por año.

El cuadro 1 muestra los resultados de cálculos basados en diferentes suposiciones acerca del ritmo medio de crecimiento natural. Las tasas de menos del 3.30 por ciento pueden descartarse de inmediato, por ser inconsistentes con los resultados de múltiples estudios sobre la migración de mexicanos hacia los Estados Unidos. Al mismo tiempo, las de más de 3.60 son dudosas debidó a que implican que alguno de los dos componentes biológicos directos del crecimiento natural ha estado más allá de los límites proba-

\section{Cuadro 1}

MEXICO: COMPONENTES DEL CRECIMIENTO DE LA POBLACION NACIONAL SEGUN DIFERENTES SUPOSICIONES ACERCA DEL CRECIMIENTO NATURAL. PERIODO INTERCENSAL 1970-80 * (MILES)

\begin{tabular}{cccc}
\hline $\begin{array}{c}\text { Tasa anual de } \\
\text { crecimiento natural } \\
\text { (por ciento) }\end{array}$ & $\begin{array}{c}\text { Crecimiento } \\
\text { natural }\end{array}$ & \multicolumn{2}{c}{ Migración internacional } \\
\hline 3.20 & $18,583.2$ & Total & Promedio anual \\
3.25 & $18,918.9$ & +597.3 & +57.7 \\
3.30 & $19,256.1$ & -261.6 & +25.3 \\
3.35 & $19,594.9$ & -75.6 & -4.3 \\
3.40 & $19,935.1$ & -714.4 & -40.0 \\
3.45 & $20,277.0$ & $-1,096.5$ & -72.9 \\
3.50 & $20,620.4$ & -1.439 .9 & -106.0 \\
3.55 & $20,965.3$ & -1.784 .8 & -139.1 \\
3.60 & $21,311.8$ & -2.131 .3 & -172.5 \\
3.65 & $21,659.9$ & -2.479 .4 & -206.0 \\
3.70 & $22,009.5$ & $-2,829.0$ & -239.6 \\
3.75 & $22,360.7$ & $-3,180.2$ & -273.4 \\
3.80 & $22,713.5$ & $-3,533.0$ & -307.3 \\
\hline
\end{tabular}

* Para estos cálculos: $\mathrm{P}_{70}=48,225.2 ; \mathrm{P}_{80}=67,405.7 ; \mathrm{y} \mathrm{n}=3777 / 365 . \mathrm{P}_{80 \mathrm{c}}=\mathrm{P}_{70}(1+\mathrm{r})^{\mathrm{n}} ; \mathrm{M}_{70-80}$ (total) $=\mathbf{P}_{800}-\mathbf{P}_{80 e} ; \mathrm{yCN}_{70-80}$ (total $)=\left(\mathbf{P}_{800}-\mathbf{P}_{70}\right)-\mathbf{M}_{70-80}$. 
bles para nuestro país durante los últimos diez años. ${ }^{3}$ Por ende, se puede concluir que el aporte real del crecimiento natural al crecimiento general de la población residente del país, probablemente se ubicó ẹ algún nivel entre el 3.3 y el 3.6 por ciento por año durante la década del setenta: nivel notablemente superior a lo previsto en la política demográfica nacional (CONAPO, 1979b).

\section{Redistribución interna de la población, 1930-1980}

Sea cual fuere la realidad acerca de los respectivos aportes del crecimiento natural medio de la población del país y de las migraciones internacionales, los datos preliminares del censo de 1980 permiten enunciar algunas conclusiones importantes acerca de la redistribución interna de la población mexicana $y$, por ende, acerca de los patrones generales de las migraciones internas. Estas conclusiones, sin embargo, serán de tipo cualitativo, y quedan muy amplios márgenes de error posible en los datos cuantitativos, a pesar de que se presentan en los cuadros de este trabajo, datos concretos con un nivel de precisión que va mucho más allá de un nivel de exactitud confiable.

Si se contempla la redistribución a largo plazo desde la óptica de las grandes aglomeraciones demográfico-regionales, es posible detectar diversas tendencias importantes, sin tomar en cuenta, por el momento, el juego entre las migraciones internas, la incidencia diferencial de la emigración neta del país en diferentes partes de su territorio las variaciones en el nivel del crecimiento vegetativo de la población (cuadro 2). ${ }^{4}$

\section{La capital nacional}

La gravitación del flujo de habitantes hacia la Ciudad de México ha sido importante durante los últimos cincuenta años $-\mathrm{y}$ se ha intensificado notablemente en cada uno de los períodos intercensales contemplados en el cuadro 2.5

A pesar de que los datos a nivel de entidad federativa (los únicos disponibles hasta ahora para 1980) no son del todo satisfactorios para este propósito, por lo menos señalan en forma adecuada las tendencias generales al respecto. Hasta 1950, el crecimiento de la Ciudad de México se

\footnotetext{
3 En términos de tasas brutas, un crecimiento medio anual del 3.60 por ciento implicaria -si se acepta la muy baja tasa de mortalidad de 7.3 defunciones por mil habitantes reportada para 1976 (CONAPO, 1979a: cuadro IV-2) - una tasa bruta de fecundidad de 43.3 nacimientos por mil habitantes, contra la de 39.3 reportada por la misma fuente para 1976.

4 Esta "regionalización" (léase, "agrupación de entidades federativas") se destina a los fines del presente trabajo exclusivamente. No se está proponiendo de ninguna manera, otra regionalización más del país para propósitos múltiples, sino una serie de agrupaciones de estados contiguos.

5 Al hablar de ciudades concretas, se hace referencia a las respectivas aglomeraciones urbanas en su totalidad (sin tomar en consideración límites politico-administrativos) - salvo en caso de que lo contrario sea señalado explícitamente.
} 
Cuadro 2

MEXICO: CRECIMIENTO DIFERENCIAL. POR GRUPOS DE ENTIIADES FEDERATIVAS EN CADA PERIODO INTERCENSAL. (1930-1980** (miles)

\begin{tabular}{|c|c|c|c|c|c|c|c|}
\hline \multirow{3}{*}{$\frac{\text { Entidad y grumo }}{\text { TOTAL NACIONAL }}$} & \multicolumn{2}{|c|}{ Poblacion } & \multicolumn{5}{|c|}{ Crecimiento diferencial } \\
\hline & 1930 & 1980 & $30-40$ & $40-50$ & $50-60$ & $60-70$ & $70-80$ \\
\hline & 16552.7 & 67405.7 & $=0.0$ & $=0.0$ & $=0.0$ & 0.0 & 0.0 \\
\hline $\begin{array}{l}\text { D.F.+EDO. DE MEX. } \\
\text { Distrito Federal } \\
\text { Estado de México }\end{array}$ & $\begin{array}{r}2219.7 \\
1229.6 \\
990.1\end{array}$ & $\frac{16919.6}{9377.3}$ & $\begin{array}{l}+268.0 \\
+297.7 \\
-\quad 29.6\end{array}$ & $\begin{array}{l}+634.5 \\
+745.1 \\
-110.6\end{array}$ & $\begin{array}{r}+749.9 \\
+738.5 \\
+\quad 11.3\end{array}$ & $\begin{array}{l}+1360.4 \\
+148.0 \\
+1212.4\end{array}$ & $\begin{array}{l}+1953.6 \\
231.0 \\
+2184.5\end{array}$ \\
\hline $\begin{array}{l}\text { PACIFICO NORTE } \\
\text { Baja Calif. Norte } \\
\text { Baja Calif. Sur } \\
\text { Sonora } \\
\text { Sinaloa }\end{array}$ & $\begin{array}{r}\frac{807.3}{48.3} \\
47.1 \\
316.3 \\
395.6\end{array}$ & $\begin{array}{r}\frac{4828.7}{1227.4} \\
221.0 \\
1498.1 \\
1882.2\end{array}$ & $\begin{array}{r}+28.9 \\
+21.6 \\
-4.4 \\
-11.4 \\
+23.1\end{array}$ & $\begin{array}{r}+139.0 \\
+123.5 \\
-\quad 6.7 \\
+\quad 32.9 \\
-10.7\end{array}$ & $\begin{array}{r}+280.7 \\
+212.7 \\
-\quad 0.9 \\
+\quad 91.7 \\
-\quad 22.8\end{array}$ & $\begin{array}{l}+293.0 \\
+152.1 \\
+\quad 15.3 \\
+\quad 16.9 \\
+108.8\end{array}$ & $\begin{array}{l}+\quad 127.3 \\
+10.8 \\
+\quad 42.1 \\
+\quad 37.6 \\
+112.0\end{array}$ \\
\hline $\begin{array}{l}\text { GOLFO Y SURESTE } \\
\text { Veracruz } \\
\text { Tabasco } \\
\text { Chiapas } \\
\text { Yucatán } \\
\text { Campeche } \\
\text { Quintana Roo }\end{array}$ & $\begin{array}{r}2612.6 \\
1377.3 \\
224.0 \\
530.0 \\
386.1 \\
84.6 \\
10.6\end{array}$ & $\begin{array}{r}10127.3 \\
5263.8 \\
1150.0 \\
2097.5 \\
1034.3 \\
371.8 \\
209.9\end{array}$ & $\begin{array}{l}+10.3 \\
+16.0 \\
+19.6 \\
+50.6 \\
+40.2 \\
+\quad 9.9 \\
+\quad 6.2\end{array}$ & $\begin{array}{l}-106.4 \\
-83.8 \\
-11.9 \\
+15.2 \\
-31.6 \\
+\quad 3.4 \\
+\quad 2.3\end{array}$ & $\begin{array}{r}-118.7 \\
-36.0 \\
+\quad 5.0 \\
-17.8 \\
-86.2 \\
+\quad 2.8 \\
+\quad 13.6\end{array}$ & $\begin{array}{r}-\quad 22.9 \\
+\quad 48.5 \\
+\quad 83.0 \\
-\quad 103.0 \\
-\quad 89.5 \\
+\quad 19.3 \\
+\quad 18.9\end{array}$ & $\begin{array}{l}-\quad 7.6 \\
-\quad 69.1 \\
+\quad 76.1 \\
-\quad 95.7 \\
-\quad 25.7 \\
+\quad 20.1 \\
+\quad 86.6\end{array}$ \\
\hline $\begin{array}{l}\text { OCCIDENTE } \\
\text { Jalisco } \\
\text { Aguascalientes } \\
\text { Zacatecas } \\
\text { Guanajuato } \\
\text { Michoacán } \\
\text { Colima } \\
\text { Nayarit }\end{array}$ & $\begin{array}{r}4313.0 \\
1255.3 \\
132.9 \\
459.0 \\
987.8 \\
1048.4 \\
61.9 \\
167.7\end{array}$ & $\begin{array}{r}13109.4 \\
4296.5 \\
504.3 \\
1144.7 \\
3045.6 \\
3094.4 \\
339.4 \\
729.5\end{array}$ & $\begin{array}{l}-214.1 \\
-72.2 \\
+\quad 3.9 \\
+\quad 20.4 \\
-126.3 \\
-\quad 62.8 \\
+\quad 5.3 \\
+\quad 17.6\end{array}$ & $\begin{array}{l}-370.6 \\
-113.6 \\
-\quad 24.0 \\
-76.1 \\
-44.0 \\
-127.7 \\
+\quad 8.9 \\
+\quad 5.9\end{array}$ & $\begin{array}{r}-148.9 \\
+76.9 \\
-11.4 \\
-83.8 \\
-64.5 \\
-75.4 \\
+12.4 \\
-\quad 3.1\end{array}$ & $\begin{array}{rr}-\quad 592.8 \\
-\quad 77.3 \\
+\quad 2.0 \\
-\quad 177.8 \\
-\quad 126.1 \\
-\quad 233.1 \\
+\quad 14.0 \\
+\quad 5.6\end{array}$ & $\begin{array}{r}-\quad 820.4 \\
-311.2 \\
+\quad 31.7 \\
-185.2 \\
-127.8 \\
-199.2 \\
+\quad 2.3 \\
-\quad 30.9\end{array}$ \\
\hline $\begin{array}{l}\text { "OTRO NORTE" } \\
\text { Nuevo Leбn } \\
\text { Chihuahua } \\
\text { Coahuila } \\
\text { Tamaulipas } \\
\text { Durango } \\
\text { San Luis Potosi }\end{array}$ & $\begin{array}{r}2673.9 \\
417.5 \\
491.8 \\
436.4 \\
344.0 \\
404.4 \\
579.8\end{array}$ & $\begin{array}{l}\frac{10714.7}{2463.5} \\
1935.1 \\
1561.0 \\
1924.9 \\
1160.3 \\
1669.9\end{array}$ & $\begin{array}{l}+162.3 \\
+45.4 \\
+40.0 \\
+\quad 32.5 \\
+\quad 50.4 \\
+\quad 3.6 \\
+\quad 9.6\end{array}$ & $\begin{array}{r}+134.2 \\
+30.5 \\
+28.0 \\
-\quad 1.7 \\
+116.4 \\
-\quad 4.7 \\
-\quad 34.3\end{array}$ & $\begin{array}{r}-65.0 \\
+76.1 \\
+80.2 \\
-68.5 \\
+51.3 \\
-92.5 \\
-111.5\end{array}$ & $\begin{array}{l}-\quad 249.4 \\
+205.0 \\
-\quad 81.6 \\
-138.4 \\
+\quad 42.6 \\
-111.4 \\
-165.6\end{array}$ & $\begin{array}{rr}-\quad 607.3 \\
+\quad 94.8 \\
-\quad 318.7 \\
+\quad 2.5 \\
-111.4 \\
-152.4 \\
-122.0\end{array}$ \\
\hline $\begin{array}{l}\text { OTROS ESTADOS } \\
\text { Morelos } \\
\text { Querêtaro } \\
\text { Guerrero } \\
\text { Hidalgo } \\
\text { Oaxaca } \\
\text { Puebla } \\
\text { Tlaxcala }\end{array}$ & $\begin{array}{r}4126.1 \\
132.1 \\
234.1 \\
641.7 \\
677.8 \\
1084.5 \\
1150.4 \\
205.5\end{array}$ & $\begin{array}{r}11706.0 \\
931.4 \\
730.9 \\
2174.2 \\
1518.2 \\
2517.5 \\
3285.3 \\
548.5\end{array}$ & $\begin{array}{l}-255.5 \\
+25.9 \\
-33.3 \\
-29.0 \\
-33.0 \\
-94.9 \\
-71.3 \\
-19.9\end{array}$ & $\begin{array}{l}-430.4 \\
+33.2 \\
-34.8 \\
-41.9 \\
-162.0 \\
-143.3 \\
-\quad 72.3 \\
-\quad 9.3\end{array}$ & $\begin{array}{l}-697.9 \\
+16.7 \\
-32.7 \\
-58.8 \\
-157.4 \\
-198.1 \\
-228.7 \\
=38.8\end{array}$ & $\begin{array}{lr}- & 788.4 \\
& 82.7 \\
-\quad 4.7 \\
-\quad 41.3 \\
-\quad 179.6 \\
-\quad 369.8 \\
-\quad 217.4 \\
-\quad 58.2\end{array}$ & $\begin{array}{r}-\quad 645.7 \\
\quad 70.3 \\
+\quad 52.3 \\
-\quad 58.5 \\
-\quad 150.5 \\
-\quad 299.5 \\
-\quad 220.5 \\
=\quad 39.4\end{array}$ \\
\hline
\end{tabular}

Cambio absoluto en el número de habitantes que no se explica por medio de la aplicación del ritmo de crecimiento nacional del periodo a la población inicial de la entidad o región - con la suposición de un saldo de cero en las migraciones internacionales. 
dio de modo fundamental pero no exclusivo en el Distrito Federal: fuera del Area Metropolitana, el resto del Estado de México, en balance, es y ha sido tradicionalmente de emigración de cierta importancia. El crecimiento de las partes de la mancha urbana de México fuera de los límites del Distrito Federal empieza a ser observable a nivel de los datos para entidades federativas enteras, en los años cincuenta; sin embargo, se intensifica mucho en términos relativos en el siguiente decenio. Con respecto a la década del setenta y a pesar del fuerte crecimiento absoluto del Distrito Federal, no parecería del todo equivocado empezar a hablar de una "fuga hacia el suburbio" semejante a la que se produjo algún tiempo antes en algunas zonas metropolitanas de los Estados Unidos.

Entre el censo de 1970 y el de 1980, la participación del Distrito Federal en el total de la población nacional bajó del 14.3 al 13.9 por ciento; mientras el Estado de México creció del 7.9 al 11.2 por ciento. Al mismo tiempo, el crecimiento del "weekendismo" entre los capitalinos (cuando no una "residencia usual" en esos estados, combinado con un departamento de "entre semana" en la ciudad o un muy largo viaje diario al trabajo), debe haber sido un factor de cierta importancia en el fuerte crecimiento que se observa para el lapso 1970-80 en los estados de Morelos y Querétaro.

\section{Pacifico Norte}

Además de la capital nacional, la región del Pacífico norte es la única que, a nivel regional mostró un ritmo de crecimiento demográfico superior al promedio nacional, durante todos los periodos intercensales entre 1930 y 1980. Este crecimiento se basó, en gran medida, en la apertura de nuevas zonas de riego y, principalmente en la última parte de este medio siglo en el crecimiento acelerado de unas cuantas ciudades fronterizas.

En cierta forma, al considerar este aspecto de la redistribución de la población mexicana, sería más conveniente pensar en una región formada por seis estados: los cuatro del grupo "Pacífico norte" del cuadro 2, más Nayarit y Colima. Hacia el norte, dentro de estos seis estados, la expansión poblacional se ha basado mayormente en la apertura de nuevas zonas de riego; $y$, hacia el sur, se trata más bien de una utilización más completa de tierras tropicales de poca altura sobre el nivel del mar. En los dos casos, el crecimiento demográfia un ritmo superior al del promedio nacional durante estos últimos cincuenta años se debió de modó preponderante a la integración al sistema productivo del pais de recursos naturales que previamente eran subaprovechados.

Tal crecimiento diferencial a nivel regional es, necesariamente, reflejo de una época transitoria en el desarrollo del país. Los recursos antes subaprovechados se explotaron al máximo o más: las posibilidades de una nueva colonización sobre estas bases se hacen cada día más escasas. El desarrollo turístico, que ha sido un factor de cierta importancia en el crecimiento regional du-

6 Más del máximo, en el sentido de una explotación "destructiva" que da lugar a la salinización en las tierras de riego en zonas áridas y a la erosión, etc., en zonas más húmedas. 
rante las últimas décadas del período considerado (aunque limitado geográficamente a unos cuantos lugares) también verá limitado su alcance.

Dentro de este grupo de seis estados, los datos demográficos (junto con los conocimientos generales acerca de eventos acaecidos durante este periodo)sugieren las siguientes conclusiones tentativas. En términos generales, se trata de una región que creció a un ritmo superior al promedio nacional en los últimos cinco períodos intercensales, pero con diferencias que se reducen en forma notable durante el periodo 1970-80. A nivel de estado, la situación es más variada, a pesar de que ninguno de los seis contemplados en el concepto más amplio de "Pacifico Norte" ha rechazado población de modo marcado en ninguna de estas décadas. Parecería que, ya en los años setenta, las nuevas oportunidades de este medio siglo se habían saturado en Sonora y en Nayarit, y que ya comenzaban a disminuir en Colima y Baja California Norte. Sinaloa y Baja California Sur - probablemente debido a factores transitorios-mostraron elevadas tasas de crecimiento respecto al conjunto del pais y hasta para este grupo de estados, durante los años setenta. La especulación que, por el momento, pareceria tener la mayor probabilidad de ser acertada es, que muy pronto la región del Pacífico Norte - definida del modo que sea - bajará del nivel de "atracción" en el crecimiento demográfico diferencial del país al nivel de "equilibrio" (cuando no hasta el de "rechazo").

\section{Golfo y Sureste}

Este es un grupo de estados con historias demográficas muy variadas que, por lo general, oscilan alrededor del promedio de crecimiento nacional. Se trata de un conjunto de estados que, como grupo o región, perdieron lugar dentro de la población nacional en los años cuarenta y cincuenta, pero que en los otros tres decenios se acercaron mucho a los promedios nacionales. En cuanto a los factores de atracción demográfica, se trata más que nada del mejor aprovechamiento de las tierras bajas del trópico-hecho que, según se reconoce cada vez más, es "problemático" en términos de la agricultura científica moderna que se desarrolló en zonas templadas- junto con los efectos de la actividad petrolera de los últimos años en algunas partes y el turismo en otras.

Por el momento, no parecen existir bases muy firmes para hacer especulaciones acerca de la futura participación de estos estados en las tendencias demográficas del país. Los avances tecnológicos en la agricultura tropical tal vez abrirían nuevos cauces para la colonización; los descubrimientos petroleros de los últimos años y su aprovechamiento obviamente han producido un impacto económico muy im portante que, a su vez, ha influido en las tendencias demográficas en forma particular, como lo demuestran diversos estudios sobre el tema (ver, por ejemplo, Allub y Michel, 1980; Lezama y Massolo, 1980; Ocampo y Ordorica, 1980; Pietri, 1980); en el caso de Quintana Roo, el desarrollo turístico ayuda para explicar su crecimiento excepcionalmente rápido.

El conjunto de estados considerados en el cuadro 2 bajo el rubro "Golfo y Sureste" completa la lista de las grandes agrupaciones poblacionales del país que, a nivel de regiones enteras, históricamente han mostrado cierta atracción o al menos equilibrio con respecto al crecimiento medio nacional. En los apartados siguientes se considerarán tres grupos de estados que, durante el último 
medio siglo, muestran una marcada disminución (como grupos), de su participación en la población nacional. Estos grupos son: "occidente", "otro norte", y "otros estados" (principalmente del centro).

\section{Occidente}

El occidente del pais, definido en base a estados enteros, es una región muy grande que tradicionalmente ha sido considerada "exportadora" de población durante el medio siglo considerado (cuadro 2; Winnie y Arroyo, 1979: capítulo II). Ya se mencionó, en relación con el Pacífico Norte, la situación especial de Colima y Nayarit: las tierras bajas de Jalisco y Michoacán también se ubican mejor dentro de los moldes generales mencionados en esa sección, de la misma manera que las tierras altas de Colima y Nayarit encajan mejor dentro del occidente.

Los primeros resultados del censo nacional de 1980 muestran una fuerte y creciente disminución de la participación de esta parte del país en la población nacional: del 24.8 por ciento en 1930 al 19.4 en 1980 . Se trata de un masivo éxodo rural que parece obedecer a la falta de nuevas oportunidades de empleo en el campo que absorba internamente el rápido crecimiento demográfico de las comunidades rurales (Guzmán y Winnie, 1980; Winnie, Guzmán y Wessman, 1980), junto con un desarrollo urbano altamente concentrado en la metrópoli regional de Guadalajara y un poco raquítico en casi todos los demás centros (Arroyo, 1978; Winnie y Arroyo, 1979: capítulo IV; Arroyo, Winnie y Velázquez, 1980; Arroyo y Velázquez, 1980). La emigración temporal o permanente hacia los Estados Unidos es una tradición bien establecida en esta región, desde hace mucho tiempo (ver, por ejemplo: Gamio, 1930; Taylor, 1933; Dagodag, 1975; Winnie, Guzmán y Hernández, 1979; Dinerman, 1978; Shadow, 1979; y Mines, 1980); los siete estados contemplados bajo este rubro en el cuadro 2 aportaron el 37.8 por ciento del total de la migración neta nacional hacia el Distrito Federal y el Estado de México en el período intercensal 1960-70 (Censo 1970, Resumen: cuadro 14). Estas migraciones hacia destinos fuera de la misma región habrían sido aún mayores en ausencia de la gran atracción ejercida por Guadalajara y algunas ciudades menores dentro del mismo Occidente (Winnie, 1978; Arroyo, Winnie y Velázquez, 1980).

Así como la región en general tiene una larga tradición de rechazo poblacional, los datos presentados en el cuadro 2 muestran algunas variaciones en este patrón nivel de estado - además de las que se esperaban debido a las situaciones especiales de Colima y Nayarit. Desde antes existían sospechas referidas a una posible subenumeración en la ciudad de Aguascalientes en 1970 (Winnie y Arroyo, 1979: 132); en base a conversaciones privadas con algunas personas que participaron en los trabajos de campo de Guadalajara en el censo de 1980 , es posible que los datos preliminares para Jalisco se vean afectados por fuertes subenumeraciones que se espera, serán corregidas en etapas posteriores del proceso censal.

\footnotetext{
7 Los datos preliminares para 1980, según se tiene entendido, son los resultados al finalizarse el conteo de la primera ronda de entrevistas en el mismo día oficial del censo. Según la prensa públi-
} 
El caso especial de Jalisco. Entre 1950 y 1970, el juego combinado del rápido crecimiento demográfico de Guadalajara y ritmos notablemente por debajo del promedio nacional para el resto del estado en su conjunto, tuvo el efecto de que Jalisco se acercara mucho a la tasa media de crecimiento del país entero en estos dos decenios: un poco más rápido durante el primero y un poco menos en el segundo, de tal manera que contó con el 6.8 por ciento de la población nacional, tanto en 1970 como veinte años antes.

La restante información disponible para el setenta - datos cuantitativos directos de la Encuesta de Hogares de la Zona Metropolitana de Guadalajara de 1972 y de la Encuesta Nacional de 1975 y 1977, así como las observaciones personales del autor y de otros científicos sociales residentes en Guadalajara durante este lapso, respecto de su crecimiento fisico y económico- sugiere que Guadalajara creció demográficamente durante los años setenta, a un ritmo semejante al que mostró en la década anterior. ${ }^{8}$

Asimismo, los datos de los cuadros 2 y 3 para los demás estados de la región no ofrecen base suficiente para suponer que el éxodo rural se haya intensificado en forma notable en esta parte del país, durante los últimos diez años.

Tentativamente, entonces, el descenso de la participación del Estado de Jalisco en la población nacional, del 6.8 por ciento en 1970 al 6.4 por ciento en 1980 , que aparece en los datos del cuadro 2 y se refleja en los del cuadro 3, debe verse con ciertas reservas hasta tanto estén disponibles los resultados definitivos del nuevo censo a nivel de municipios.

El caso especial de Aguascalientes. En contraste con la situación del estado de Jalisco, los datos preliminares del censo de 1980 sugieren un crecimiento demográfico excepcionalmente rápido en este estado durante la década del setenta. A reserva de ver los resultados definitivos del censo de 1980 al nivel de municipios, el conteo preliminar al nivel del estado entero refuerza la especulación antes mencionada acerca de una posible subenumeración importante en la ciudad de Aguascalientes en 1970.

Algunas especulaciones tentativas de tipo más general. Es evidente a nivel regional, que el occidente definido en términos de los siete estados señalados en los cuadros 2 y 3, continuó entre 1970 y 1980, su patrón histórico de crecimiento demográfico a un ritmo inferior al del promedio nacional.

ca, la omisión en esa ronda era de algo semejante al diez por ciento del territorio; y, según conversaciones privadas con participantes en el levantamiento de ese primer dia, importantes áreas enteras de la Zona Metropolitana de Guadalajara fueron omitidas en su totalidad. Supuestamente, las labores censales después de ese primer día de enumeración captaron la información para la población originalmente omitida, la cual será integrada en los datos definitivos. En base a estas consideraciones, se debe suponer provisoriamente que el conteo para Jalisco reportado en el cuadro 2 crecerá notablemente en los datos definitivos.

8 Este ritmo sería geométrico, en cuanto al crecimiento natural de la población al inicio del decenio, y aritmético en cuanto al volumen de la migración neta. En base a esta suposición y datos para el período intercensal 1960-70, podemos esperar una inmigración neta de entre 330000 y 390000 para el periodo intercensal 1970-80. Al mismo tiempo, el crecimiento natural (incluyendo el de los nuevos migrantes) habrá sido de unos 552000 al ritmo de los años sesenta 0429000 a un ritmo medio del 20 por ciento más bajo (aproximadamente, 2.5 por ciento anual). Tales límites sugieren que la población total de la Zona Metropolitana de Guadalajara a la fecha del censo de 1980 debe haber sido de alrededor de 2210000 o 2400000 . 
En este primer intento de análisis e interpretación de los resultados iniciales del censo de 1980 por estados enteros, ya se mencionaron Colima, Nayarit y Jalisco como casos especiales debido a sus características demostrables; y el último de ellos junto con Aguascalientes por posibles problemas de datos. Los otros tres estados del grupo - Zacatecas, Guanajuato y Michoacánse acercaron un poquito más al ritmo de crecimiento nacional en los años setenta que en los sesenta, aunque no mucho (cuadro 3 ).

Este último hecho, junto con todo lo mencionado acerca de Aguascalientes y observaciones sobre el terreno en muchas comunidades rurales en el transcurso del setenta, conducen a las siguientes especulaciones a nivel hipotético acerca de la región en general.

El bajo ritmo de crecimiento de la población rural que ha sido característico de gran parte de esta extensa región, probablemente se haya generalizado aún más durante la década del setenta; posiblemente, incluso, se haya intensificado en algunas comunidades donde ya era muy notable en los sesentas.

Asimismo, y a nivel hipotético hasta tanto haya información más completa y definitiva, parece muy probable que durante los últimos diez años, el desarrollo de algunas ciudades "secundarias" y "menores" que ya se destacaban como centros de atracción inicipiente para sus respectivas zonas en 1970, hayan continuado su tendencia anterior e incluso a un ritmo mayor y en un grado tal que hayan sido un factor importante en el descenso del ritmo de pérdida demográfica relativa de sus respectivos estados. Sería muy arriesgado especular ahora acerca de cuáles de estos centros urbanos habrán aportado más en este sentido durante el setenta: algunos como Fresni1lo, Aguascalientes, León y otros en Guanajuato, etc. es casi seguró que figurarán dentro de la lista, (ver Winnie y Arroyo, 1979; capítulo IV; Arroyo y Velázquez, 1980; Pi-Sunyer, 1973).

\section{"Otro Norte"}

Esta región es, fundamentalmente, la zona del país que se sitúa al oriente de la Sierra Madre Occidental, en la cual la escasez de lluvias impone límites severos a la agricultura que no sea de riego. Dentro de ella, y en notable contraste con el Pacífico Norte, las tierras más fácilmente irrigables ya contaban con sus sistemas de riego mucho antes de los setenta si no desde antes del inicio de mediados del siglo considerado en este trabajo.

Otra vez, dos estados dentro del grupo (ahora, de seis estados) comparten características demográficas con otra región. En este caso, Durango y San Luis Potosí encajan mejor en los moldes de Occidente que en los de los demás estados norteños.

Nuevo León creció más rápidamente que el país en general, en cada periodo intercensal a partir de 1930. Monterrey indudablemente ha sido el factor principal durante este lapso, pero el desarrollo cítrico de la zona de Montemorelos y el crecimiento de algunos centros urbanos menores como Linares también deben haber influido, al menos en forma transitoria. Como en el caso de Guadalajara, se trata de una de las tres principales metrópolis del país que obviamente creció mucho durante la última década. 
Cuadro 3

MEXICO: INDICES DE CRECIMIENTO DEMOGRÁFICO DIFERENCIAL* POR REGIONES Y ESTADOS

1930-1980

\begin{tabular}{|c|c|c|c|c|c|}
\hline \multirow{2}{*}{ Entidad y grupo } & \multicolumn{5}{|c|}{ Indice } \\
\hline & $1930-40$ & $1940-50$ & $1950-60$ & $1960-70$ & $1970-80$ \\
\hline TOTAL NACIONAL & $\underline{100}$ & $\underline{100}$ & 100 & $\underline{100}$ & $\underline{100}$ \\
\hline $\begin{array}{l}\text { D.F. + EDO. MEX. } \\
\text { Distrito Federal } \\
\text { Estado de México }\end{array}$ & $\begin{array}{r}110 \\
120 \\
97\end{array}$ & $\begin{array}{r}\frac{117}{132} \\
93\end{array}$ & $\begin{array}{l}\frac{112}{118} \\
101\end{array}$ & $\begin{array}{l}\frac{115}{102} \\
146\end{array}$ & $\begin{array}{l}\frac{113}{98} \\
141\end{array}$ \\
\hline $\begin{array}{l}\text { PACIFICO NORTE } \\
\text { Baja Calif. Norte } \\
\text { Baja Calif. Sur } \\
\text { Sonora } \\
\text { Sinaloa }\end{array}$ & $\begin{array}{r}\frac{103}{138} \\
92 \\
97 \\
105\end{array}$ & $\begin{array}{r}\frac{111}{219} \\
90 \\
107 \\
98\end{array}$ & $\begin{array}{r}\frac{114}{169} \\
99 \\
113 \\
97\end{array}$ & $\begin{array}{l}\frac{110}{121} \\
114 \\
102 \\
109\end{array}$ & $\begin{array}{r}\frac{103}{101} \\
124 \\
98 \\
106\end{array}$ \\
\hline $\begin{array}{l}\text { GOLFO Y SURESTE } \\
\text { Veracruz } \\
\text { Tabasco } \\
\text { Chiapas } \\
\text { Yucatan } \\
\text { Campeche } \\
\text { Quintana Roo }\end{array}$ & $\begin{array}{r}\frac{100}{99} \\
107 \\
108 \\
91 \\
90 \\
149\end{array}$ & $\begin{array}{r}\frac{97}{96} \\
97 \\
102 \\
94 \\
103 \\
109\end{array}$ & $\begin{array}{r}\frac{98}{99} \\
101 \\
99 \\
88 \\
102 \\
137\end{array}$ & $\begin{array}{r}100 \\
101 \\
112 \\
94 \\
89 \\
108 \\
127\end{array}$ & $\begin{array}{r}\frac{100}{99} \\
107 \\
96 \\
98 \\
106 \\
170\end{array}$ \\
\hline $\begin{array}{l}\text { OCCIDENTE } \\
\text { Jalisco } \\
\text { Aguascalientes } \\
\text { zacatecas } \\
\text { Guanajuato } \\
\text { Michoacan } \\
\text { Colima } \\
\text { Nayarit }\end{array}$ & $\begin{array}{r}96 \\
95 \\
102 \\
104 \\
89 \\
95 \\
107 \\
109\end{array}$ & $\begin{array}{r}\frac{94}{94} \\
89 \\
90 \\
97 \\
92 \\
109 \\
102\end{array}$ & $\begin{array}{r}98 \\
103 \\
96 \\
91 \\
96 \\
96 \\
108 \\
99\end{array}$ & $\begin{array}{r}\frac{94}{98} \\
101 \\
84 \\
95 \\
91 \\
106 \\
101\end{array}$ & $\begin{array}{r}\frac{94}{93} \\
107 \\
86 \\
96 \\
94 \\
101 \\
96\end{array}$ \\
\hline $\begin{array}{l}\text { "OTRO NORTE" } \\
\text { Nuevo Lebn } \\
\text { Chihuahua } \\
\text { Coahuila } \\
\text { Tamaulipas } \\
\text { Durango } \\
\text { San Luis Potosi }\end{array}$ & $\begin{array}{r}105 \\
109 \\
107 \\
106 \\
112 \\
91 \\
99\end{array}$ & $\begin{array}{r}103 \\
104 \\
103 \\
100 \\
119 \\
99 \\
96\end{array}$ & $\begin{array}{r}99 \\
108 \\
107 \\
93 \\
105 \\
89 \\
90\end{array}$ & $\begin{array}{r}197 \\
114 \\
95 \\
89 \\
103 \\
89 \\
89\end{array}$ & $\begin{array}{r}95 \\
104 \\
86 \\
100 \\
95 \\
88 \\
93\end{array}$ \\
\hline $\begin{array}{l}\text { OTROS ESTADOS } \\
\text { Morelcs } \\
\text { Queretaro } \\
\text { Guerrero } \\
\text { Hidalgo } \\
\text { Oaxaca } \\
\text { Puebla } \\
\text { Tlaxcala }\end{array}$ & $\begin{array}{r}95 \\
1 \frac{95}{16} \\
88 \\
88 \\
96 \\
93 \\
95 \\
92\end{array}$ & $\begin{array}{r}93 \\
1 \frac{114}{89} \\
89 \\
84 \\
91 \\
96 \\
97\end{array}$ & $\begin{array}{r}91 \\
105 \\
92 \\
92 \\
86 \\
90 \\
90 \\
90\end{array}$ & $\begin{array}{r}92 \\
115 \\
99 \\
99 \\
87 \\
84 \\
92 \\
88\end{array}$ & $\begin{array}{r}95 \\
.108 \\
108 \\
108 \\
91 \\
89 \\
94 \\
93\end{array}$ \\
\hline
\end{tabular}

* El Indice en cada caso es igual al porcentaje de la poblacion total del pais que tenía el area al final del período, dividido por el porcentaje semejante que tenía al inicio del mismo período -multiplicado por 100 . 
Los tres estados restantes de este grupo - Chihuahua, Coahuila y Tamaulipas - también mostraron ritmos de crecimiento demográfico superiores al promedio nacional, al principio del período (1930-40). En primer lugar, Coahuila descendió hasta el nivel del promedio nacional en los años cuarenta, para alcanzar niveles notablemente más bajos entre 1950 y 1970; Chihuahua continuó por encima del promedio hasta 1960 y Tamaulipas se ubicó en una posición algo superior en 1960-70.

A comienzos del período 1930-1980, el "progresismo" del Norte que ya databa de mucho tiempo antes, puede haber sido importante tanto en la apertura de nuevas zonas de riego como en la urbanización. Ultimamente -en los años setenta- los seis estados de este grupo, en conjunto, mostraron un índice de crecimiento demográfico comparativo casi idéntico al del grupo de siete estados de Occidente. A pesar de la gran atención concedida a las ciudades fronterizas en los últimos años (ver, por ejemplo, Bustamante, 1979; Bustamante Lemus, 1980; Cámara, 1979; y Revel-Monroz y Bataillon, 1977) y el rápido crecimiento de Monterrey, este "Otro Norte" ya se asemeja al Occidente en el sentido de ser una región "exportadora" de población.

\section{"Otros Estados" (principalmente del Centro)}

Los estados del Centro del país, excepción hecha de la aglomeración metropolitana de la Capital Nacional, tienen una historia tan larga y hasta más pronunciada como "exportadores" de población que Occidente.

Sería repetitivo considerar cada uno de los siete estados incluidos en este grupo en los cuadros 2 y 3 por separado. Ya se mencionaron consideraciones especiales referidas a Morelos y Querétaro en la sección correspondiente a la Ciudad de México. Los otros cinco, sin excepción, crecieron a ritmos inferiores al promedio nacional en las cinco décadas a partir de 1930.

Con excepción de Guerrero, se trata de partes del país que están "llenas" de población desde hace mucho tiempo. Es probable que los nuevos avances tecnológicos que se introduzcan en el agro en el futuro, reduzcan las necesidades de mano de obra en el campo, así como que el fuerte crecimiento vegetativo de su propia población durante los últimos 20-30 años garantice una creciente demanda de oportunidades para "ganarse la vida" durante por lo menos el resto del presente siglo.

\section{Componentes de la redistribución interna}

Las variaciones que se observan en la tasa general de crecimiento demográfico en las diferentes regiones y estados del país, son el resultado de los efectos combinados de las variaciones en el ritmo de crecimiento vegetativo y el saldo neto de las migraciones de todo tipo. En un primer nivel de análisis, estos dos componentes pueden desglosarse en fecundidad y mortalidad, en el primer caso, y movimientos internos e internacionales, en el segundo. 


\section{Crecimiento natural}

Existe muy poca (si acaso alguna), relación entre las tasas vitales reportadas en 1976 en base a las estadisticas vitales (CONAPO, 1979b: cuadro IV-2) y las tasas de crecimiento demográfico intercensal de l período 1970-80. A nivel de las 32 entidades federativas, las correlaciones de las tasas vitales con el crecimiento intercensal no son significativas en absoluto, no importa que se considere el crecimiento natural en forma global, o sus dos componentes por separado, la relación mayor es de $\mathrm{r}=0.27$.

Variaciones en el ritmo real del crecimiento vegetativo de la población indudablemente existen y tendrán alguna influencia. Sin embargo, parecería que la suposición con mayor probabilidad de acercarse a la verdad en el estudio de la redistribución de la población mexicana entre 1970 y 1980 es, que las variaciones en los niveles del crecimiento natural tuvieron una influencia comparativamente pequeña y que las migraciones deben haber constituido el factor principal.9

\section{Tendencias en el impacto de las migraciones internas}

Los datos definitivos del censo de 1980 incluirán información directa sobre los movimientos interestatales del último decenio. Estos indudablemente variarán de modo significativo respecto de las primeras estimaciones, basadas en el cambio en el número total de habitantes que se presentan en el cuadro 2. Como ya se mencionó, las variaciones en el ritmo de crecimiento vegetativo y en la incidencia de las migraciones internacionales también influyen en estos diferenciales; pero el factor dominante es el saldo interestatal en las migraciones internas.

Aun en ausencia de los datos directos sobre las migraciones internas - que pronto saldrán del procesamiento del censo de 1980 — podrían justificarse algunas conclusiones generales basadas en los primeros datos sobre el número de habitantes por entidad federativa.

Sólo una de las seis agrupaciones de entidades contempladas en los cuadros 2 y 3 -la que representa la Capital Nacional, a nivel del país entero- puede ser calificada como de "atracción migratoria" en el último decenio. Además, no hay nada que indique que la tendencia histórica a la concentración de la población nacional debido a las migraciones internas haya cambiado radicalmente durante los últimos diez años.

Paralelamente, la época de gran atracción migratoria del Pacífico Norte parece haber llegado a su fin en la década del setenta ya que, en términos de estas agrupaciones se ubica, junto con el Golfo y el Sureste, alrededor del equilibrio migratorio. Además, los datos para estos estados y los otros estados fronterizos sugieren que es posible que en los últimos años se haya exa-

9 Los datos del censo de 1970 sobre el número de hijos nacidos vivos por mujer, en edades reproductivas, (Resumen, cuadro 30) tampoco ayudan en la explicación de las variaciones en el crecimiento demográfico general que se observan entre los diferentes estados para el período 1970-80. Las respectivas correlaciones son muy bajas: +0.24 para mujeres de 25-29 años de edad, +0.11 para las de $30-34$ y +0.04 para las de $45-49$. 
gerado la importancia de las ciudades fronterizas en las migraciones nacionales.

En la última década otras partes del pais, en términos generales, han experimentado emigraciones netas a nivel de estado. A pesar de las importantes variaciones que se observan entre estados concretos, a nivel regional la emigración ha sido fuerte.

\section{Implicaciones para la política demográfica del país}

El análisis de los primeros resultados del censo de 1980 y su relación con otros datos, ha dado lugar a algunas conclusiones provisorias que parecen revistir gran importancia para la evaluación y la readaptación de las politicas demográficas prevalecientes. Como mínimo, estas conclusiones sugieren que se realicen análisis más profundos una vez que estén disponibles los resultados definitivos del censo de 1980.

En esta sección se comentará la política demográfica oficial del país, en términos de lo que parece ser la experiencia de años recientes en relación con a) el crecimiento natural y b) las políticas referentes a la migración.

\section{Crecimiento natural}

Los primeros resultados del censo de 1980, en comparación con los de censos anteriores, las estadísticas vitales, y otros, despiertan muchas dudas acerca de la posibilidad de que las últimas estimaciones del ritmo de crecimiento natural de la población mexicana sean acertadas - $-\mathrm{y}$ aun más dudas con respecto a la factibilidad de cumplir con la meta de un crecimiento medio anual del 2.5 por ciento para $1982-$. El promedio para los años setenta no puede haber sido muy inferior al 3.3 por ciento anual, si en realidad no fue superior a ese nivel.

\section{Migración: política de retención}

Mientras más estudios se hacen al respecto, menores parecen ser las posibilidades de producir una mayor "retención migratoria" en las comunidades rurales mexicanas propiamente dichas (ver Winnie, Arroyo y Guzmán, 1980: citas 1234-1253). Si los recursos locales disponibles para la economía rural ya han sido totalmente aprovechados (según la tecnologia existente), el elevado crecimiento vegetativo tendrá que ser contrapesado con traslados poblacionales hacia otras partes: en conjunto, los cambios tecnológicos que se adopten ahora no aumentarán las posibilidades de retener más población en el lugar sino que por el contrario, en definitiva tenderán más a desplazar mano de obra con capital y a asignar cualquier aumento que se produzca en la productividad por día-hombre de trabajo, a receptores (comerciantes, financiadores o dueños de capital) fuera de la comunidad local.

En estas circunstancias, la "política de retención" expresada a nivel de estados enteros como es el caso de la Política Demográfica Regional (CONAPO, 1979a: 79-92) tiene que convertirse en forma casi obligada, en una reorienta- 
ción de la emigración rural, que actualmente se dirige a otros estados, hacia centros urbanos alternativos dentro del estado de origen.

\section{Migración: política de reorientación}

La política general se destina a reducir la "excesiva" concentración demográfica en unas cuantas metrópolis muy grandes y, dentro de ella, la política de reorientación

"... se fundamenta en cambiar el destino de una parte de las corrientes migratorias, de las entidades que más expulsan población hacia las áreas metropolitanas y ciudades mayores de México. La reorientación tendría como punto final otras entidades federativas, con capacidad en su estructura económica para absorber nuevos migrantes, además de tener la característica de ser entidades de equilibrio, puesto que expulsan y atraen población en los mismos volúmenes." (CONAPO, 1979a:79-80).

La conveniencia de este enfoque que está basado en los saldos migratorios de estados enteros, es muy dudosa. Al menos en los ocho estados para los cuales el CISE* elaboró estudios sociodemográficos en 1980 (los siete de Occidente, más Sinaloa), el "equilibrio" que se da a nivel del estado en algunos casos, es fundamentalmente el resultado neto del juego - a nivel interno del estado- entre emigraciones fuertes de algunas partes que son de rechazo, por un lado, e inmigraciones netas (que varian entre saldos cerca de cero hasta bastante fuertes en otras partes que son de equilibrio o atracción), por el otro. Las partes de estos ocho estados que fueron "de equilibrio" o "atracción" en los años sesenta, en la gran mayoría de los casos (Puerto Vallarta es la excepción principal, ver Martínez, 1977), lo fueron porque absorbieron más migrantes procedentes de orígenes cercanos dentro de la misma región que los que perdieron debido a su propia emigración hacia destinos más distantes (CISE, 1980). Sin contar con estudios similares para los otros estados "de equilibrio", es muy probable que su "equilibrio" estatal tenga una composición semejante.

Estas consideraciones sugieren que sería más acertado definir la "política de reorientación" en términos distintos y de modo tal que casi se fusionara con la "politica de retención" definida en términos de estados enteros. La redefinición de la "política de reorientación", vista en este sentido, "reorientaría" la emigración de las zonas de fuerte rechazo poblacional a nivel subestatal que actualmente se dirige a las grandes metrópolis, hacia centros urbanos secundarios o menores dentro de sus propias regiones. Muchos centros con los niveles mencionados ya muestran una cierta capacidad de absorción demográfica de esta naturaleza: el enfoque propuesto implicaría un esfuerzo espe-

* CISE: Centro de Investigaciones Sociales y Económicas. Facultad de Economía, Universidad de Guadalajara, México. 
cial (acompañado, por supuesto, de ciertas inversiones públicas) para reforzar las tendencias hacia el rápido crecimiento urbano, ya incipiente en un número reducido de tales centros, en cada región. ${ }^{10}$

\section{Migraciones: politica de reubicación}

La política de reubicación (CONAPO, 1979a: 80, 97-100) es más importante por sus posibles aportes a la implementación de la política de reorientación definida en los términos propuestos en la sección anterior, que por su incidencia numérica en los grandes patrones de la migración. Dirigida hacia la promoción del desarrollo urbano de un número reducido de centros cuidadosamente seleccionados, esta parte de la política demográfica nacional (adecuadamente coordinada, por supuesto, con otros programas oficiales en favor del desarrollo de los mismos centros) tiene la posibilidad de ser un elemento importante dentro de "un paquete" de medidas para reorientar las migraciones que actualmente se dirigen hacia las grandes metrópolis.

\section{Conclusiones generales}

Como se ha visto, los primeros datos del nuevo censo nacional muestran algunos cambios importantes en los grandes patrones de redistribución de la población nacional que prevalecieron en la década anterior - si no en varias décadas anteriores.

En términos generales, las conclusiones del presente trabajo señalan la continuación de la tendencia hacia una mayor concentración de la población del país en la Capital Nacional (como se esperaba) y, además, una tendencia bastante notable a la reducción de las diferencias interregionales entre otras partes de la República, en su ritmo de crecimiento demográfico comparativo. Ya en la década del setenta, las dos entidades que representan la Ciudad de México, formaron la única unidad en este nivel que puede calificarse como "de atracción", mientras apenas otras dos estuvieron cerca del "equilibrio" y tres más fueron "de rechazo". Además, se ha demostrado que las estimaciones más recientes del ritmo de crecimiento natural casi seguramente están por debajo de las reales y en un grado significativo.

Las conclusiones concretas presentadas en este trabajo tienen importantes implicaciones para las políticas demográficas oficiales del país. En principio, estas implicaciones - aun en el actual nivel de conclusiones provisorias- señalan la necesidad urgente de confrontar las metas referidas al crecimiento natural con los hechos reportados por el censo de 1980, a fin de efectuar los reajustes convenientes y, sin esperar más información censal, iniciar un proce-

10 En opinión del autor, sería muy equivocado aplicar medidas parejas o proporcionales al tamaño actual en todos los centros urbanos. La concentración de los recursos para estos propósitos en un pequeño número de centros cuidadosamente seleccionados, podría aportar en forma importante, no solamente a la reorientación de las migraciones internas en los términos bajo consideración, sino también a llenar un "vacío" en las estructuras urbanas del país. (Para algunas consideraciones adicionales al respecto, ver Winnie, 1974.) 
so de reformulación de las políticas relativas a la migración de modo de hacerlas conformar mejor a las realidades que vive el país en el inicio de la década del ochenta. 